\title{
Temperature modulation of fatty acid profiles for biofuel production in nitrogen deprived Chlamydomonas reinhardtii
}

\author{
Gabriel O. James ${ }^{a}$, Charles H. Hocart ${ }^{b}$, Warwick Hillier ${ }^{a}$, G. Dean Price ${ }^{a}$, Michael A. Djordjevic ${ }^{a, *}$ \\ a Plant Science Division, Research School of Biology, College of Medicine, Biology and Environment, The Australian National University, Canberra, ACT 0200, Australia \\ ${ }^{\mathrm{b}}$ Mass Spectrometry Facility, Research School of Biology, College of Medicine, Biology and Environment, The Australian National University, Canberra, ACT 0200, Australia
}

\section{H I G H L I G H T S}

- Algae often produce higher levels of polyunsaturated fatty acids than is desirable for biodiesel.

- Temperature shifts can alter the fatty acid profile of storage lipids under nitrogen starvation.

- $32^{\circ} \mathrm{C}$ was the optimal temperature for fatty acid content and composition for biodiesel production.

- Carbohydrate and lipid storage were both affected by shifting temperature.

- Environmental factors can manipulate algal feedstocks potentially reducing the refining cost.

\section{A R T I C L E I N F O}

\section{Article history:}

Received 14 August 2012

Received in revised form 22 September 2012

Accepted 25 September 2012

Available online 11 October 2012

\section{Keywords:}

Chlamydomonas reinhardtii

Algae

Biofuel

Fatty acid

Temperature

\begin{abstract}
A B S T R A C T
This study investigated the changes in the fatty acid content and composition in the nitrogen-starved Chlamydomonas reinhardtii starchless mutant, BAF-J5, grown at different temperatures.

The optimal temperature for vegetative growth under nitrogen sufficient conditions was found to be $32{ }^{\circ} \mathrm{C}$. Shifting temperature from 25 to $32^{\circ} \mathrm{C}$, in conjunction with nitrogen starvation, resulted in BAFJ5 storing the maximum quantity of fatty acid (76\% of dry cell weight). Shifting to temperatures lower than $25^{\circ} \mathrm{C}$, reduced the total amount of stored fatty acid content and increased the level of desaturation in the fatty acids. The optimal fatty acid composition for biodiesel was at $32{ }^{\circ} \mathrm{C}$. This study demonstrates how a critical environmental factor, such as temperature, can modulate the amount and composition of fatty acids under nitrogen deprivation and reduce the requirement for costly refining of biofuels.
\end{abstract}

(c) 2012 Elsevier Ltd. All rights reserved.

\section{Introduction}

The mass cultivation of microalgae as a biomass feedstock for liquid biofuels is being assessed worldwide. Microalgae have high photosynthetic productivity and growth rates compared to terrestrial plants and can accumulate energy rich compounds such as polysaccharides and lipids. Microalgal lipids are of particular interest for nutrition (Mansour et al., 2005) and as a replacement for petroleum based transportation fuels such as diesel and jet fuel (Lee Chang et al., 2012). Therefore, a greater understanding of the biological processes and environmental factors that influence the storage of these metabolites is required. For mass cultivation in the open, seasonal changes in light and temperature have the potential to significantly affect algal growth and modulate productivity. These environmental factors could directly impact the economic viability for industrial-scale biofuel production.

\footnotetext{
* Corresponding author. Tel.: +61 (2) 61253088.

E-mail address: michael.djordjevic@anu.edu.au (M.A. Djordjevic).
}

The eukaryotic green alga Chlamydomonas reinhardtii is an important model for the study of photosynthesis (Grossman, 2000) and lipid metabolism in microalgae (Lohr et al., 2005). The availably of a sequenced genome with mature annotation (Merchant et al., 2007) and metabolic databases (Lopez et al., 2011) enables high-throughput techniques like transcriptional, proteomic and metabolomic profiling to be applied to understanding lipid metabolism. The availability of strain collections and mutant libraries also facilitates the elucidation of lipid metabolism in microalgae (Boyle et al., 2012; Miller et al., 2010; Nguyen et al., 2011; Wang et al., 2011). The metabolic consequences of changes to environmental conditions or induced by functionally removing a gene or disrupting its regulation may have unanticipated consequences. Therefore it is important to directly identify and quantify the metabolites to fully understand the biology.

Chlamydomonas reinhardtii normally stores carbon in starch granules and lipid droplets when nitrogen deprived (Ball et al., 1990; Miller et al., 2010; Moellering and Benning, 2010; Msanne et al., 2012; Nguyen et al., 2011). However, in the low-starch 
(sta1-1) (Ball et al., 1991) and starchless mutants (sta6) (Zabawinski et al., 2001), carbon is redirected from starch to lipid biosynthesis when starved of nitrogen (Fan et al., 2011; Goodson et al., 2011; James et al., 2011; Wang et al., 2009). These mutants are defective in the key regulatory enzyme of starch biosynthesis, ADP-glucose Pyrophosphorylase (AGPase). This shift in carbon metabolism from starch to lipid biosynthesis and storage has also been observed in higher plants with reduced levels of AGPase (Sanjaya et al., 2011). In C. reinhardtii, photoautotrophic (Msanne et al., 2012) and photoheterotrophic growth under low and high-light (Li et al., 2010) have been investigated under nitrogen deprivation. However, there has been no study on the influence of shifts in growth temperature on lipid biosynthesis under nitrogen deprivation.

The optimisation of growth temperature is an important parameter as the composition of lipids in thylakoid membranes and photosystems of both microalgae and cyanobacteria are temperature sensitive, and acclimatise accordingly (see review by Los and Murata, 2004). Photosystem (PS) II and to a lesser extent PSI, are damaged at low or high temperatures (Allakhverdiev et al., 2008). The degree of fatty acid unsaturation in the chloroplasts is likely to be an important response mechanism for maintaining activity by adjusting the membrane fluidity of the photosystems as temperature changes. Increases in temperature lead to increased saturation of the fatty acids and conversely saturation is reduced as temperature falls (see review by Los and Murata, 2004).

While lipid quantity is important from a productivity point of view, the composition is also critical as the acyl chain lengths and their degrees of unsaturation are key properties that determine biodiesel oxidative stability and performance properties (Knothe, 2011). High levels of polyunsaturated fatty acids (high susceptibility to oxidation) or saturated lipids (raise cloud points and viscosity) are undesirable (see reviews by Knothe, 2011; Schenk et al., 2008). Optimising the fatty acid profile can reduce the extent of costly refining for biofuels (Merchant et al., 2012). Therefore, microalgae that produce predominantly monounsaturated fatty acids with low levels of saturated and polyunsaturated fatty acids would be preferable for the production of biodiesel.

Chlamydomonas reinhardtii low-starch and starchless mutants have been shown to accumulate high levels of lipid when switched to nitrogen deprivation under standard laboratory growth conditions, however, they generally produce higher proportions of polyunsaturated fatty acids than is ideal for biodiesel production. To date, no assessment has been made of the effect of temperature on lipid synthesis or composition in these strains during the switch to nitrogen deprivation. In this study, the optimal temperature for vegetative growth was determined and, crucially, the effect of temperature transition on fatty acid content and composition under nitrogen deprivation were measured. We tested the hypothesis that raising the temperature of microalgal cultures towards the maximum tolerated for growth under nitrogen deprivation would lower levels of polyunsaturated lipids, and hence produce an improved fatty acid profile for biodiesel production.

\section{Methods}

\subsection{Strains and growth conditions}

Chlamydomonas reinhardtii starchless mutant BAF-J5 (cw15 arg7-7 nit1 nit2 sta6-1::ARG7) was compared to the reference wild-type strain cc-125 ( $m t+a g g-1+$ nit1 nit2) under nitrogen sufficient and deficient conditions as described previously by James et al., 2011. In brief, the strains were grown photoheterotrotrophically in $250 \mathrm{~mL}$ flasks containing $125 \mathrm{~mL}$ of the nitrogen sufficient Tris/Acetate/Phosphate (TAP) medium (Gorman and Levine, 1965) on an orbital shaker $(100 \mathrm{rpm})$ with continuous illumination $\left(100 \pm 5 \mu \mathrm{mol}\right.$ photons $\left.\mathrm{m}^{-2} \mathrm{~s}^{-1}\right)$ at $25^{\circ} \mathrm{C}$ with standard air $\mathrm{CO}_{2}$ levels.

\subsection{Temperature effects on vegetative growth with sufficient nitrogen}

To determine growth rates an aliquot $(2 \mathrm{~mL})$ of mid-log phase culture grown at $25{ }^{\circ} \mathrm{C}$ was inoculated into TAP medium $(125 \mathrm{~mL})$ and then transferred to a illuminated growth chamber set at either $17,25,32,35$ or $38^{\circ} \mathrm{C}$ and grown until stationary phase (determined by absorbance $\mathrm{Abs}_{750}$ ). Optical density $\left(\mathrm{Abs}_{750}\right.$ ) was measured with an Ultrospec $2000 \mathrm{UV} /$ visible spectrophotometer (Pharmacia Biotech). Cell doubling time $\left(T_{\mathrm{d}}\right)$ was calculated using the formula below, where $\mu$ is the slope of the linear regression of a plot of the log of optical density over linear time (h):

$T_{\mathrm{d}}=\frac{\log (2)}{\mu}$

\subsection{Effects of temperature shifts on growth under nitrogen deprivation}

For nitrogen deprived growth studies, stationary culture $(100 \mathrm{~mL})$ grown at $25^{\circ} \mathrm{C}$ was centrifuged $\left(800 \mathrm{~g}, 10 \mathrm{~min}, 25^{\circ} \mathrm{C}\right)$ and the pellet washed with nitrogen deficient medium $(50 \mathrm{~mL}$, TAP-N) before inoculating into TAP-N medium $(200 \mathrm{~mL})$. All cultures were washed and inoculated at an initial absorbance of $\mathrm{Abs}_{750}=0.8 \pm 0.06$ (SD) and grown at either $17,25,32,35$ or $38{ }^{\circ} \mathrm{C}$ for four days.

\subsection{Estimation of total carbohydrate and lipid}

After four days of growth in nitrogen deficient medium, cells $(0.5 \mathrm{~mL})$ were pelleted $(16,000 \mathrm{~g}, 5 \mathrm{~min})$ and resuspended in water. The samples were dried under nitrogen purge and the lipid and carbohydrate content was estimated using Fourier Transformed Infrared Spectroscopy (FTIR) as previously described by James et al. (2011).

\subsection{Fatty acid analysis by gas chromatography mass spectrometry (GC/MS)}

After four days of growth in nitrogen deficient medium, algal cultures (100 mL, 40-90 mg dry cell weight, DCW), were centrifuged (3000g, $20 \mathrm{~min}$ at $25^{\circ} \mathrm{C}$ ) and the cell pellet was frozen in liquid nitrogen and freeze-dried (Bench Top $\mathrm{K}$ series freeze dryer, VirTis, USA) for four days at $-100{ }^{\circ} \mathrm{C}$ and $10-20$ mTorr.

Fatty acid methyl esters (FAMEs) were prepared and analysed by GC/MS as previously described by James et al. (2011). Quantitative analysis of the FAMEs was performed using Xcalibur software (version 1.4, Thermo Electron Corporation, USA) against the internal standard, heptadecanoic acid (Sigma-Aldrich).

\section{Results}

\subsection{Growth characteristics under nitrogen replete conditions}

The growth of $C$. reinhardtii wild-type cc-125 and the starchless mutant BAF-J5 were assessed over a range of temperatures in TAP medium. Both strains grew logarithmically at $17,25,32$ and $35^{\circ} \mathrm{C}$ but growth rate and maximum cell density were inhibited at $38^{\circ} \mathrm{C}$ (Fig. 1(a) and (b)). This was reflected in the calculated doubling times $\left(T_{\mathrm{d}}\right)$ which indicated maximum growth rates occurred at 32 and $35^{\circ} \mathrm{C}$ (Table 1 ). After 3 days at $38^{\circ} \mathrm{C}$, BAF-J5 ceased cell division and the culture collapsed (Fig. 1), however, culture col- 


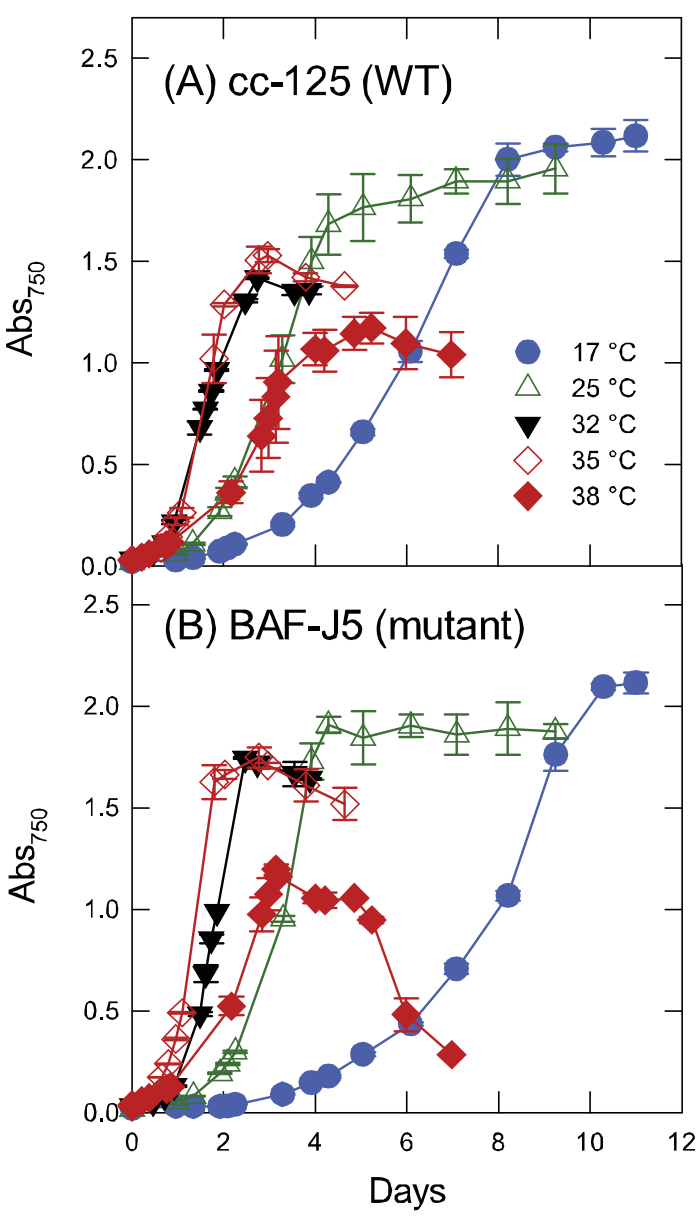

Fig. 1. Vegetative growth experiments in nitrogen replete medium at 17, 25, 32, 35 or $38^{\circ} \mathrm{C}$. Starter cultures of (a) cc-125 and (b) BAF-J5 were grown at $25^{\circ} \mathrm{C}$ and then used to inoculate fresh nitrogen replete medium and were grown until stationary phase in growth chambers set at the specified temperatures. Data as means \pm SD $(n=4)$.

Table 1

Nitrogen replete doubling times of cc-125 and BAF-J5 at different temperatures in TAP medium.

\begin{tabular}{lrr}
\hline & \multicolumn{1}{c}{ cc-125 } & \multicolumn{1}{c}{ BAF-J5 } \\
\hline $17{ }^{\circ} \mathrm{C}$ & $23.1 \pm 1.1$ & $25.7 \pm 0.3$ \\
$25^{\circ} \mathrm{C}$ & $10.7 \pm 0.6$ & $11.5 \pm 0.6$ \\
$32^{\circ} \mathrm{C}$ & $7.6 \pm 0.1$ & $7.2 \pm 0.1$ \\
$35^{\circ} \mathrm{C}$ & $8.1 \pm 0.1$ & $8.6 \pm 0.2$ \\
$38^{\circ} \mathrm{C}$ & $17.5 \pm 2.8$ & $15.5 \pm 0.7$ \\
\hline
\end{tabular}

Data expressed as cell doubling time $\left(T_{\mathrm{d}}\right)(\mathrm{h})$.

Means $n=4$ replicates, errors $=$ standard deviation.

lapse was not observed in cc-125. At $17^{\circ} \mathrm{C}$, growth rates were greatly decreased for both cc-125 and BAF-J5, being about $30 \%$ of the maximum observed at $32{ }^{\circ} \mathrm{C}$ (Table 1 ).

3.2. The effect of temperature shift, under nitrogen deprivation, on growth and carbon storage

The effect of shifting temperature under nitrogen deprivation on the growth of both strains was assessed (Fig. 2). As expected, non-logarithmic growth occurred at all temperatures with both strains (James et al., 2011).

Using FTIR, the wild-type strain, cc-125, was seen to store excess carbon primarily as starch with only low levels of lipid. The highest levels of carbohydrate were observed at 25,32 and $35^{\circ} \mathrm{C}$

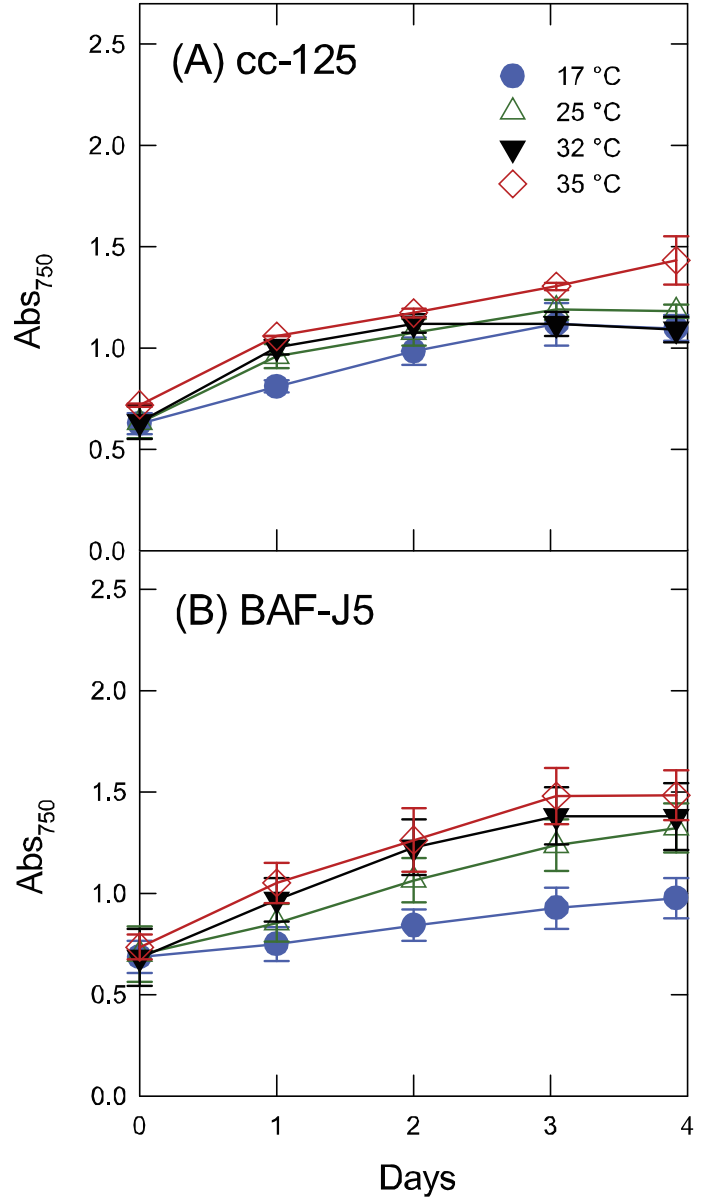

Fig. 2. Changes in growth under nitrogen deprivation after shifting cultures to temperatures of $17,25,32$ or $35^{\circ} \mathrm{C}$. Starter cultures of (a) cc-125 and (b) BAF-J5 were grown to stationary phase at $25^{\circ} \mathrm{C}$, then diluted in fresh nitrogen-free medium and grown for four days in growth chambers set at the specified temperatures. Data as means $\pm \operatorname{SD}(n=4)$.

(Supplementary Fig. 1(a)). Carbohydrate storage was lowest at 17 and $38{ }^{\circ} \mathrm{C}$. Similar levels of lipid were observed at 17, 25, 32 and $35^{\circ} \mathrm{C}$. In the starchless mutant, BAF-J5, low levels of starch storage, and high levels of lipid storage were observed after four days of nitrogen limitation and at each transition temperature except $38^{\circ} \mathrm{C}$ (Supplementary Fig. 1(b)). The maximal levels of lipid content in whole cells were produced at 25 and $32^{\circ} \mathrm{C}$, with a reduction in storage at 17,35 and $38^{\circ} \mathrm{C}$ (Supplementary Fig. 1(b)). These semi-quantitative FTIR measurements of lipid content were confirmed by quantitative measurements of fatty acid content by GC/MS analysis (Table 2).

\subsection{Quantification of fatty acids}

After four days of nitrogen starvation, fatty acids were quantified by GC/MS following extractive methylation to fatty acid methyl esters (FAMEs; Table 2). The major fatty acids were 16:0, 18:0. $18: 1^{\Delta 9}, 18: 1^{\Delta 11}, 18: 2^{\Delta 9,12}$ and $18: 3^{\Delta 9,12,15}$, as previously identified by James et al., 2011. The total amount of hydrolysed fatty acids extracted from the wild type strain, cc-125, did not vary significantly with shifts in temperature (Table 2 and Fig. 3(a)). By contrast, fatty acid production in the starchless mutant, BAF-J5, was higher and ranged from $55.6 \% \mathrm{DCW}$ after shifting to $17^{\circ} \mathrm{C}$ to a maximum $76.4 \% \mathrm{DCW}$ at $32{ }^{\circ} \mathrm{C}$ before decreasing to $74.2 \% \mathrm{DCW}$ at $35^{\circ} \mathrm{C}$ (Table 2, Fig. 3(a)). The total fatty acid production at each shift of temperature was consistent with FTIR analysis (Fig 3(b)). At 
Table 2

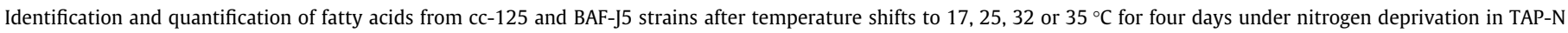
medium.

\begin{tabular}{|c|c|c|c|c|c|c|c|c|}
\hline & \multicolumn{4}{|l|}{ cc-125 } & \multicolumn{4}{|l|}{ BAF-J5 } \\
\hline & $17^{\circ} \mathrm{C}$ & $25^{\circ} \mathrm{C}$ & $32{ }^{\circ} \mathrm{C}$ & $35^{\circ} \mathrm{C}$ & $17^{\circ} \mathrm{C}$ & $25^{\circ} \mathrm{C}$ & $32{ }^{\circ} \mathrm{C}$ & $35^{\circ} \mathrm{C}$ \\
\hline $16: 0$ & 3.28 & 3.18 & 3.14 & 4.06 & 15.22 & 16.83 & 18.60 & 22.55 \\
\hline${ }^{a} 16: 1 \Delta^{7 t}$ & 0.05 & 0.04 & 0.04 & 0.05 & 0.64 & 1.59 & 4.13 & 4.39 \\
\hline${ }^{a} 16: 1 \Delta^{7 c}$ & 0.10 & 0.02 & 0.05 & 0.02 & 0.21 & 0.26 & 0.39 & 0.23 \\
\hline $16: 2 \Delta^{7,10}$ & 0.19 & 0.25 & 0.29 & 0.28 & 0.49 & 0.79 & 0.82 & 0.64 \\
\hline $16: 3 \Delta^{7,10,13}$ & 0.11 & 0.13 & 0.21 & 0.16 & 0.06 & 0.06 & 0.06 & 0.07 \\
\hline $16: 3$ & 0.42 & 0.33 & 0.21 & 0.10 & 2.22 & 1.94 & 2.05 & 1.71 \\
\hline $16: 4 \Delta^{4,7,10,13}$ & 1.02 & 0.94 & 0.94 & 0.79 & 2.29 & 2.21 & 1.90 & 1.87 \\
\hline $18: 0$ & 0.39 & 0.44 & 0.50 & 0.74 & 1.05 & 2.02 & 2.61 & 2.75 \\
\hline $18: 1 \Delta^{9}$ & 0.77 & 0.53 & 0.48 & 0.41 & 8.52 & 13.74 & 16.33 & 12.25 \\
\hline $18: 1 \Delta^{11}$ & 0.83 & 1.15 & 1.24 & 1.58 & 3.46 & 4.91 & 5.86 & 6.18 \\
\hline $18: 2 \Delta^{9,12}$ & 2.04 & 1.73 & 1.39 & 1.36 & 9.99 & 12.01 & 10.84 & 8.49 \\
\hline $18: 3 \Delta^{5,9,12}$ & 1.04 & 1.22 & 1.55 & 1.32 & 2.83 & 3.90 & 5.57 & 6.49 \\
\hline $18: 3 \Delta^{9,12,15}$ & 2.78 & 2.48 & 2.09 & 1.77 & 8.34 & 7.71 & 6.83 & 6.80 \\
\hline $\mathrm{b}_{20} 0,18: 4 \Delta^{5,9,12,15}$ & 0.17 & 0.23 & 0.26 & 0.23 & 0.31 & 0.33 & 0.41 & 0.53 \\
\hline $20: 1 \Delta^{9}$ & 0.01 & 0.01 & 0.01 & 0.00 & 0.31 & 0.33 & 0.41 & 0.53 \\
\hline FAME Total & 13.21 & 12.68 & 12.41 & 12.54 & 55.63 & 68.29 & 76.40 & 74.15 \\
\hline
\end{tabular}

Data expressed as a percentage of dry cell weight (dcw). Means $n=4$ replicates, standard deviations $\leqslant 4.86 \%$

${ }^{a}$ cis or trans geometry of bond determined from relative retention time.

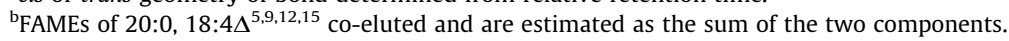

the lowest transition temperature $17^{\circ} \mathrm{C}$, the fatty acid content for cc-125 was relatively constant at $13.2 \%$. However, for BAF-J5, the fatty acid level was almost three quarters of the maximum achieved at $32{ }^{\circ} \mathrm{C}$ (Table 2, Fig. 3(a)).

The accumulation and relative proportions of saturated, unsaturated and total fatty acids was assessed for both strains at the different transition temperatures (Table 2, Fig. 3). In BAF-J5, monounsaturated fatty acids increased with temperature until $32{ }^{\circ} \mathrm{C}$ (Fig. 3(b)). The relative proportions of saturated fatty acid for both strains remained essentially constant at 17,25 and $32{ }^{\circ} \mathrm{C}$ but increased sharply at $35^{\circ} \mathrm{C}$ (Fig. 3(c)). The relative proportion of polyunsaturated fatty acids in both strains also declined consistently but above $32{ }^{\circ} \mathrm{C}$, the wild type strain, cc-125, declined much more sharply (Fig. 3(d)). For the starchless mutant, BAF-J5, this resulted in a maximisation of the ratios of mono- to poly-unsaturated fatty acids (Fig. 3(e)) and mono- to saturated fatty acid at $32{ }^{\circ} \mathrm{C}$ (Fig. 3(f)).

\section{Discussion}

\subsection{Growth characteristics}

The optimal growth temperature for vegetative growth in a nitrogen sufficient medium was found to be $32^{\circ} \mathrm{C}$, although similar growth was also achieved at $35^{\circ} \mathrm{C}$ (Fig. 1). At $38^{\circ} \mathrm{C}$, both strains grew poorly and reached lower optical density, indicating that the maximum tolerable temperature for growth occurs between 35 and $38{ }^{\circ} \mathrm{C}$. At $38{ }^{\circ} \mathrm{C}$ the culture of BAF-J5 collapsed after 5 days (Fig. 1(b)), whereas cc-125 did not collapse (Fig. 1(a)). BAF-J5 contains multiple mutations affecting not only starch biosynthesis, but also the flagella and the cell wall synthesis (Zabawinski et al., 2001) and this may account for its high temperature sensitivity. At $17^{\circ} \mathrm{C}$, the maximum growth rate of both cc-125 and BAF-J5 was reduced by approximately $60 \%$. The shifting of cultures to a range of temperatures under nitrogen deprivation resulted in an expected slow non-logarithmic growth for both strains (Fig. 2) as previously observed by James et al., 2011.

\subsection{Fatty acid content}

Under nitrogen deprivation, shifting temperature to $32{ }^{\circ} \mathrm{C}$ resulted in BAF-J5 storing up to $76 \%$ of its DCW as fatty acids. At $35^{\circ} \mathrm{C}$, a similar level of total fatty acid also accumulated (74\% DCW). This is in accord with our previous work at $25^{\circ} \mathrm{C}$, when BAF-J5 was found to increase its fatty acid content from $14 \%$ to $65 \%$ DCW following nitrogen starvation (James et al., 2011). Under nitrogen deprivation, the total fatty acid content of cc-125 remained constant at $\sim 12 \%$ DCW over the range of transition temperatures from 17 to $35^{\circ} \mathrm{C}$. At $17^{\circ} \mathrm{C}$, the fatty acid content of BAF-J5 was reduced by about a third relative to that at $32^{\circ} \mathrm{C}$. This suggests that lipid storage, as measured by fatty acid content, is less affected by the transition to nitrogen starvation (30\% decrease) and low temperature $\left(17^{\circ} \mathrm{C}\right)$ than is cell division in nutrient sufficient media at low temperature, as evidenced by a $60 \%$ increase in the cell doubling time. Carbohydrate and lipid storage were both affected by switching temperature, indicating precursor supply is rate limiting for carbon storage under nitrogen starvation (Supplementary Fig. 1). This is consistent with a recent report that showed acetate availability controls fatty acid synthesis and lipid storage in C. reinhardtii under nitrogen starvation (Fan et al., 2012). Recent studies have shown that fatty acid biosynthesis is up-regulated when $C$. reinhardtii wild-type and starchless strains are grown photoheterotrophically and nitrogen deprived (Fan et al., 2011; Moellering and Benning, 2010; Wang et al., 2011).

\subsection{Fatty acid unsaturation and membrane fluidity}

This study shows that under nitrogen starvation, the level of fatty acid unsaturation is influenced by a temperature shift. For BAF-J5 at $35^{\circ} \mathrm{C}$, the observed increase in saturated fatty acids was accompanied by a decrease in monounsaturated and polyunsaturated fatty acids relative to other temperatures tested. This may reflect adaptive changes in fatty acid metabolism needed to adjust to temperature regimes near the maximum tolerated by $C$. reinhardtii. A similar response was also recorded for cc-125 at $35{ }^{\circ} \mathrm{C}$ compared to $32{ }^{\circ} \mathrm{C}$ : a sharp decrease in polyunsaturated fatty acids was accompanied by a sharp increase in saturated lipids. This may be linked with a response requirement to maintain a certain level of membrane fluidity (Los and Murata, 2004). As temperature rises, the level of polyunsaturated fatty acids declines and concomitantly there is a rise in monounsaturated fatty acids. This is consistent with other studies that show exposure to a lower growth temperature increases polyunsaturated fatty acid production in 

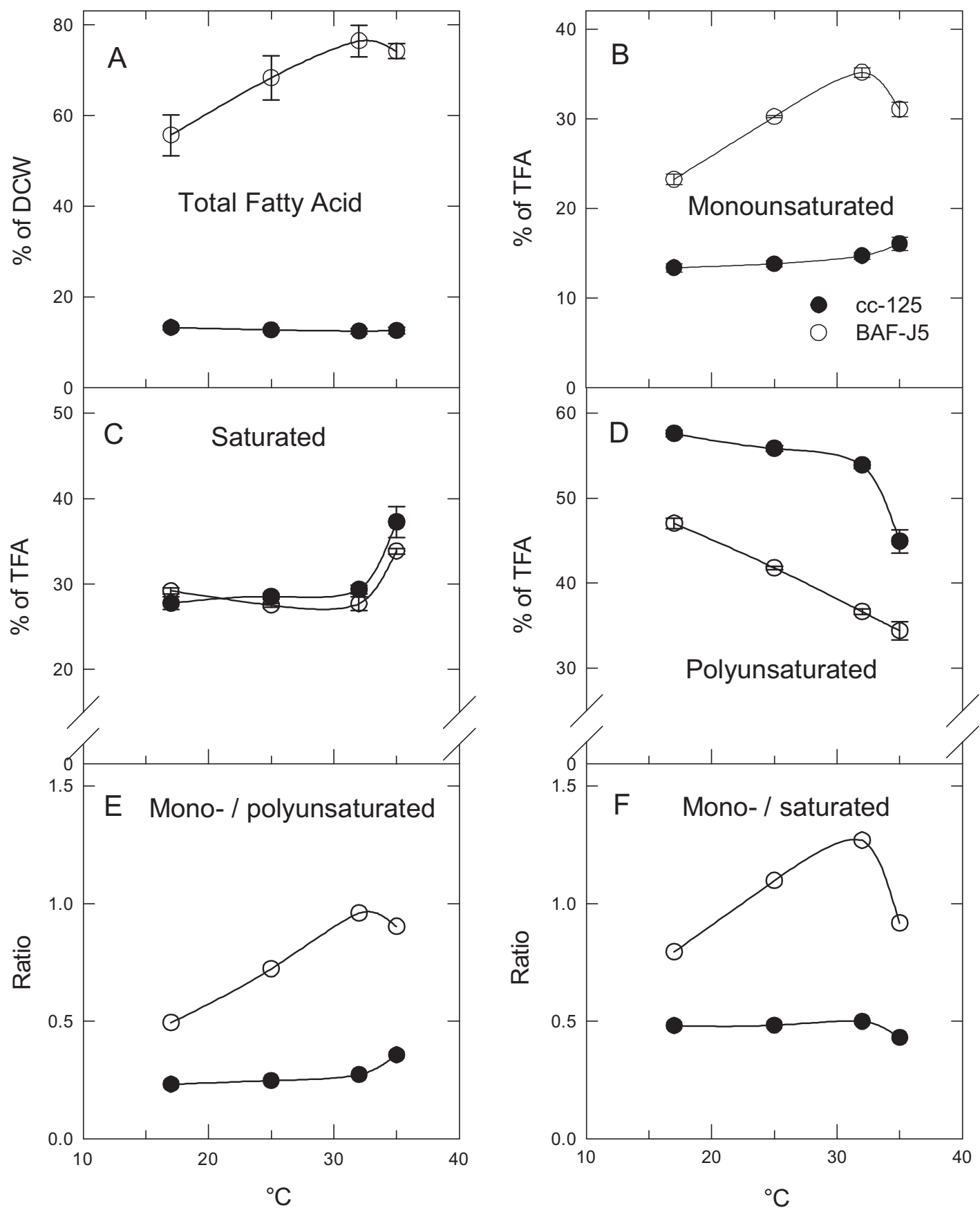

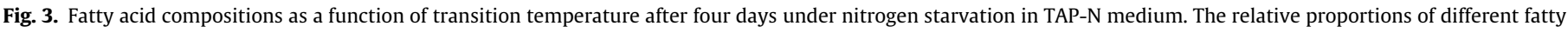

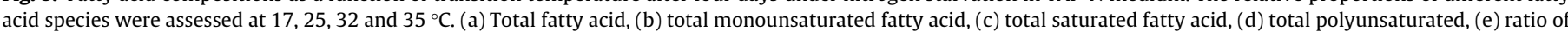
total monounsaturated/total polyunsaturated fatty acid and (f) ratio of total monounsaturated/total saturated. Data as means \pm SD $(n=4)$.

cyanobacteria (Mironov et al., 2012), algae (Lynch and Thompson, 1984) and higher plants (Zheng et al., 2011).

In both cc-125 and BAF-J5, the highly unsaturated fatty acids $16: 4^{\Delta 4,7,10,13}$ and $18: 3^{\Delta 9,12,15}$ which are predominantly associated with the chloroplast lipid classes monogalactosyldiacylglycerol (MGDG) and sulfoquinovosyl diacylglycerol (SQDG) (Giroud et al., 1988), decreased as temperature increased. This is consistent with previous work that showed unsaturation of chloroplast lipids enhanced the thermal stability of photosystem II and growth at low temperature (Sato et al., 1996). This suggests the desaturation of fatty acids in storage lipids may be regulated in part by the same temperature acclimatisation mechanism as the thylakoid membrane lipids in the chloroplast. Conversely, the levels of the extra- plastidic fatty acids $18: 3^{\Delta 5,9,12}$ and $18: 4^{\Delta 5,9,12,15}$ associated with diacylglyceryl-trimethylhomoserine (DGTS) and phosphatidylethanolamine (PtdEtn) (Giroud et al., 1988) increased along with the saturated and mono-unsaturated fatty acids as temperature increased.

It is well known that growth temperature affects the fatty acid composition of photosynthetic microorganisms. The level of fatty acid unsaturation is linked to membrane fluidity and temperature (Los and Murata, 2004). Membrane fluidity decreases as temperature decreases and fatty acid desaturation increases to compensate for the decreased membrane fluidity, conversely the opposite occurs as temperature rises (Los and Murata, 2004). The C. reinhardtii $h f-9$ mutant which is deficient in plastidic $\omega-6$ fatty acid desatur- 
ase, has been reported to contain decreased levels of the chloroplast fatty acids $16: 4^{\Delta 4,7,10,13}$ and $18: 3^{\Delta 9,12,15}$, and showed reduced photosystem activities (Sato et al., 1995). Mutant $h f-9$ also displayed decreased growth at low temperature and increased growth at high temperature suggesting that chloroplast lipid unsaturation contributes to temperature acclimatisation (Sato et al., 1996). It is clear from both our results and the literature that growth temperature can be used to manipulate the composition of fatty acids for biofuel production.

Recently, several fatty acid desaturases have been found to be associated with lipid droplets (Nguyen et al. 2011). In a separate study, Miller et al. (2010) found that three desaturase genes; plastid acyl-ACP $\Delta 9$-desaturase, and the microsomal $\Delta 12$-desaturase and $\Delta 5$-desaturase were up-regulated in the dw15.1 (cw15) strain when deprived of nitrogen. Although there is no information on changes in the regulation of these desaturases with temperature, these desaturases may serve as targets for metabolic engineering to improve the fatty acid profile for biodiesel production.

\subsection{Fatty acid profile for biodiesel}

Biodiesel properties and compliance with regulatory specifications (ASTM D6751 in the USA and EN 14214 in Europe) is dependent on the fatty acid composition. Microalgal oils often contain high levels of saturated and polyunsaturated fatty acids which are likely to have poor biodiesel properties; low viscosity and high cloud point for the saturated FAMEs and low oxidative stability for the polyunsaturated FAMEs (Knothe, 2011). Recently, a Chlamydomonas sp. isolate was reported to have a fatty acid composition that meets the European Standard (EN 14214) for biodiesel (Wu et al., 2012). In our study, $32^{\circ} \mathrm{C}$ was found to be the optimum temperature for vegetative growth and for maximum content of fatty acids during nitrogen deprived growth. This temperature also provided the most favourable fatty acid profile for biodiesel production achievable for $C$. reinhardtii. The highest level of monounsaturated fatty acid was achieved, with concomitantly reduced levels of the polyunsaturated fatty acids and little change in the levels of saturated fatty acids (Fig. 3). At the highest transition temperature of $35^{\circ} \mathrm{C}$, the fatty acid profile changed to a less ideal composition for biodiesel, as saturated fatty acids increased and monounsaturated fatty acids decreased when nitrogen deprived. This demonstrates how the control of temperature can be applied to manipulate the fatty acid composition of microalgae to improve biofuel properties and performance. The desaturases responsible for the biosynthesis of specific polyunsaturated fatty acids may represent targets for genetic engineering to further improve the fatty acid composition for biodiesel production.

\section{Conclusions}

The lipid content of Chlamydomonas reinhardtii starchless mutant BAF-J5 was enhanced from $65 \%$ (at $25^{\circ} \mathrm{C}$ ) to $76 \%$ of dry cell weight following a temperature switch and onset of nitrogen deprivation at $32{ }^{\circ} \mathrm{C}$. Specific polyunsaturated fatty acids were found to respond differently to changes in transition temperature and it is postulated that this could be due to their association with organelle-specific lipid classes. This study offers an insight into how environmental factors can be used to manipulate the quantity and composition of algal fatty acids to yield a biofuel feedstock with a reduced requirement for costly refining.

\section{Acknowledgements}

This research was financially supported by an Australian Postgraduate Award provided by the Australian Government, Depart- ment of Innovation, Industry, Science and Research. Elizabeth Harris and Steven Ball are thanked for generously providing the wild-type strain cc-125, and for the starch mutant strain BAF-J5, respectively.

\section{Appendix A. Supplementary data}

Supplementary data associated with this article can be found, in the online version, at http://dx.doi.org/10.1016/j.biortech.2012.09. 090 .

\section{References}

Allakhverdiev, S.I., Kreslavski, V.D., Klimov, V.V., Los, D.A., Carpentier, R., Mohanty P., 2008. Heat stress: an overview of molecular responses in photosynthesis. Photosynthesis Research 98 (1-3), 541-550.

Ball, S., Marianne, T., Dirick, L., Fresnoy, M., Delrue, B., Decq, A., 1991. A Chlamydomonas reinhardtii low-starch mutant is defective for 3phosphoglycerate activation and orthophosphate inhibition of ADP-glucose pyrophosphorylase. Planta 185 (1), 17-26.

Ball, S.G., Dirick, L., Decq, A., Martiat, J.C., Matagne, R., 1990. Physiology of starch storage in the monocellular alga Chlamydomonas reinhardtii. Plant Science 66 (1), 1-9.

Boyle, N.R., Page, M.D., Liu, B., Blaby, I.K., Casero, D., Kropat, J., Cokus, S.J., HongHermesdorf, A., Shaw, J., Karpowicz, S.J., Gallaher, S.D., Johnson, S., Benning, C., Pellegrini, M., Grossman, A., Merchant, S.S., 2012. Three acyltransferases and nitrogen-responsive regulator are implicated in nitrogen starvation-induced triacylglycerol accumulation in Chlamydomonas. Journal of Biological Chemistry 287 (19), 15811-15825.

Fan, J., Andre, C., Xu, C., 2011. A chloroplast pathway for the de novo biosynthesis of triacylglycerol in Chlamydomonas reinhardtii. FEBS Letters 585 (12), 1985-1991.

Fan, J., Yan, C., Andre, C., Shanklin, J., Schwender, J., Xu, C., 2012. Oil accumulation is controlled by carbon precursor supply for fatty acid synthesis in Chlamydomonas reinhardtii. Plant and Cell Physiology 53 (8), 1380-1390.

Giroud, C., Gerber, A., Eichenberger, W., 1988. Lipids of Chlamydomonas reinhardtii. Analysis of molecular species and intracellular site(s) of biosynthesis. Plant and Cell Physiology 29 (4), 587-595.

Goodson, C., Roth, R., Wang, Z.T., Goodenough, U., 2011. Structural correlates of cytoplasmic and chloroplast lipid body synthesis in Chlamydomonas reinhardtii and stimulation of lipid body production with acetate boost. Eukaryotic Cell 10 (12), 1592-1606.

Gorman, D.S., Levine, R.P., 1965. Cytochrome f and plastocyanin: their sequence in the photosynthetic electron transport chain of Chlamydomonas reinhardtii. Proceedings of the National Academy of Sciences 54 (6), 1665-1669.

Grossman, A.R., 2000. Chlamydomonas reinhardtii and photosynthesis: genetics to genomics. Current Opinion in Plant Biology 3 (2), 132-137.

James, G.O., Hocart, C.H., Hillier, W., Chen, H., Kordbacheh, F., Price, G.D., Djordjevic M.A., 2011. Fatty acid profiling of Chlamydomonas reinhardtii under nitrogen deprivation. Bioresource Technology 102 (3), 3343-3351.

Knothe, G., 2011. A technical evaluation of biodiesel from vegetable oils vs. algae. Will algae-derived biodiesel perform? Green Chemistry 13 (11), 3048-3065.

Lee Chang, K.J., Dunstan, G.A., Abell, G.C.J., Clementson, L.A., Blackburn, S.I., Nichols, P.D., Koutoulis, A., 2012. Biodiscovery of new Australian thraustochytrids for production of biodiesel and long-chain omega-3 oils. Applied Microbiology and Biotechnology 93 (5), 2215-2231.

Li, Y., Han, D., Hu, G., Dauvillee, D., Sommerfeld, M., Ball, S., Hu, Q., 2010 Chlamydomonas starchless mutant defective in ADP-glucose pyrophosphorylase hyper-accumulates triacylglycerol. Metabolic Engineering 12 (4), 387-391.

Lohr, M., Im, C.-S., Grossman, A.R., 2005. Genome-based examination of chlorophyll and carotenoid biosynthesis in Chlamydomonas reinhardtii. Plant Physiology 138 (1), 490-515.

Lopez, D., Casero, D., Cokus, S.J., Merchant, S.S., Pellegrini, M., 2011. Algal functional annotation tool: a web-based analysis suite to functionally interpret large gene lists using integrated annotation and expression data. BMC Bioinformatics, 12

Los, D.A., Murata, N., 2004. Membrane fluidity and its roles in the perception of environmental signals. Biochimica et Biophysica Acta - Biomembranes 1666 (1-2), 142-157.

Lynch, D.V., Thompson, G.A., 1984. Chloroplast phospholipid molecular species alterations during low temperature acclimation in dunaliella. Plant Physiology 74 (2), 198-203.

Mansour, M.P., Frampton, D.M.F., Nichols, P.D., Volkman, J.K., Blackburn, S.I., 2005 Lipid and fatty acid yield of nine stationary-phase microalgae: applications and unusual C24-C28 polyunsaturated fatty acids. Journal of Applied Phycology 17 (4), 287-300

Merchant, S.S., Kropat, J., Liu, B., Shaw, J., Warakanont, J., 2012. TAG, you're it! Chlamydomonas as a reference organism for understanding algal triacylglycerol accumulation. Current Opinion in Biotechnology 23 (3), 352-363.

Merchant, S.S., Prochnik, S.E., Vallon, O., Harris, E.H., Karpowicz, S.J., Witman, G.B., Terry, A., Salamov, A., Fritz-Laylin, L.K., Maréchal-Drouard, L., Marshall, W.F., Qu, L.H., Nelson, D.R., Sanderfoot, A.A., Spalding, M.H., Kapitonov, V.V., Ren, Q., Ferris, P., Lindquist, E., Shapiro, H., Lucas, S.M., Grimwood, J., Schmutz, J., Grigoriev, I.V., Rokhsar, D.S., Grossman, A.R., Cardol, P., Cerutti, H., Chanfreau, G., 
Chen, Cognat, V., Croft, M.T., Dent, R., Dutcher, S., Fernández, E., Fukuzawa, H. González-Ballester, D., González-Halphen, D., Hallmann, A., Hanikenne, M. Hippler, M., Inwood, W., Jabbari, K., Kalanon, M., Kuras, R., Lefebvre, P.A., Lemaire, S.D., Lobanov, A.V., Lohr, M., Manuell, A., Meier, I., Mets, L., Mittag, M., Mittelmeier, T., Moroney, J.V., Moseley, J., Napoli, C., Nedelcu, A.M., Niyogi, K. Novoselov, S.V., Paulsen, I.T., Pazour, G., Purton, S., Ral, J.P., Riaño-Pachón, D.M., Riekhof, W., Rymarquis, L., Schroda, M., Stern, D., Umen, J., Willows, R., Wilson, N., Zimmer, S.L., Allmer, J., Balk, J., Bisova, K., Chen, C.J., Elias, M., Gendler, K., Hauser, C., Lamb, M.R., Ledford, H., Long, J.C., Minagawa, J., Page, M.D., Pan, J., Pootakham, W., Roje, S., Rose, A., Stahlberg, E., Terauchi, A.M., Yang, P., Ball, S. Bowler, C., Dieckmann, C.L., Gladyshev, V.N., Green, P., Jorgensen, R., Mayfield, S., Mueller-Roeber, B., et al., 2011. The Chlamydomonas genome reveals the evolution of key animal and plant functions. Science 318 (5848), 245-251.

Miller, R., Wu, G., Deshpande, R.R., Vieler, A., Gartner, K., Li, X., Moellering, E.R., Zauner, S., Cornish, A.J., Liu, B., Bullard, B., Sears, B.B., Kuo, M.H., Hegg, E.L., Shachar-Hill, Y., Shiu, S.H., Benning, C., 2010. Changes in transcript abundance in chlamydomonas reinhardtii following nitrogen deprivation predict diversion of metabolism. Plant Physiology 154 (4), 1737-1752.

Mironov, K.S., Sidorov, R.A., Trofimova, M.S., Bedbenov, V.S., Tsydendambaev, V.D. Allakhverdiev, S.I., Los, D.A., 2012. Light-dependent cold-induced fatty acid unsaturation, changes in membrane fluidity, and alterations in gene expression in synechocystis. Biochimica et Biophysica Acta (BBA) - Bioenergetics 1817 (8), 1352-1359.

Moellering, E.R., Benning, C., 2010. RNA interference silencing of a major lipid droplet protein affects lipid droplet size in Chlamydomonas reinhardtii. Eukaryotic Cell 9 (1), 97-106.

Msanne, J., Xu, D., Konda, A.R., Casas-Mollano, J.A., Awada, T., Cahoon, E.B., Cerutti, H., 2012. Metabolic and gene expression changes triggered by nitrogen deprivation in the photoautotrophically grown microalgae Chlamydomonas reinhardtii and Coccomyxa sp. C-169. Phytochemistry 75, 50-59.

Nguyen, H.M., Baudet, M., Cuine, S., Adriano, J.M., Barthe, D., Billon, E., Bruley, C., Beisson, F., Peltier, G., Ferro, M., Li-Beisson, Y., 2011. Proteomic profiling of oil bodies isolated from the unicellular green microalga Chlamydomonas reinhardtii: with focus on proteins involved in lipid metabolism. Proteomics 11 (21), 4266-4273.
Sanjaya, Durrett, T.P., Weise, S.E., Benning, C., 2011. Increasing the energy density of vegetative tissues by diverting carbon from starch to oil biosynthesis in transgenic Arabidopsis. Plant Biotechnology Journal 9 (8), 874-883.

Sato, N., Sonoike, K., Kawaguchi, A., Tsuzuki, M., 1996. Contribution of lowered unsaturation levels of chloroplast lipids to high temperature tolerance of photosynthesis in Chlamydomonas reinhardtii. Journal of Photochemistry and Photobiology B: Biology 36 (3), 333-337.

Sato, N., Tsuzuki, M., Matsuda, Y., Ehara, T., Osafune, T., Kawaguchi, A., 1995 Isolation and characterization of mutants affected in lipid metabolism of Chlamydomonas reinhardtii. European Journal of Biochemistry 230 (3), 987-993.

Schenk, P., Thomas-Hall, S., Stephens, E., Marx, U., Mussgnug, J., Posten, C., Kruse, O., Hankamer, B., 2008. Second generation biofuels: high-efficiency microalgae for biodiesel production. Bioenergy Research 1 (1), 20-43.

Wang, H., Alvarez, S., Hicks, L.M., 2011. Comprehensive comparison of iTRAQ and label-free LC-based quantitative proteomics approaches using two Chlamydomonas reinhardtii strains of interest for biofuels engineering. Journal of Proteome Research 11 (1), 487-501.

Wang, Z.T., Ullrich, N., Joo, S., Waffenschmidt, S., Goodenough, U., 2009. Algal lipid bodies: stress induction, purification, and biochemical characterization in wildtype and starchless Chlamydomonas reinhardtii. Eukaryotic Cell 8 (12), 18561868.

Wu, L.F., Chen, P.C., Huang, A.P., Lee, C.M., 2012. The feasibility of biodiesel production by microalgae using industrial wastewater. Bioresource Technology $113,14-18$.

Zabawinski, C., Van den Koornhuyse, N., D'Hulst, C., Schlichting, R., Giersch, C., Delrue, B., Lacroix, J.M., Preiss, J., Ball, S., 2001. Starchless mutants of Chlamydomonas reinhardtii lack the small subunit of a heterotetrameric ADP-glucose pyrophosphorylase. Journal of Bacteriology 183 (3), 1069-1077.

Zheng, G., Tian, B.O., Zhang, F., Tao, F., Li, W., 2011. Plant adaptation to frequent alterations between high and low temperatures: remodelling of membrane lipids and maintenance of unsaturation levels. Plant, Cell \& Environment 34 (9), 1431-1442. 